\title{
Relation Longueur-Poids Pour 15 Espèces de Poissons Exploitées dans la Lagune Ebrié, Côte d'Ivoire (Afrique de l'uest)
}

\section{Boni Laurence, PhD}

Unité de Formation et de Recherche des Sciences de l'Homme et de la Société, Département de Géographie, Université Félix Houphouët Boigny,

République de Côte d'Ivoire

Nobah Céline Sidonie Koco, Maître de Conférences

Ecole Normale Supérieure d'Abidjan, Département des Sciences et Technologie, Section Science de la Vie et de la Terre, Abidjan, Côte d'Ivoire

Konan Kouadio Justin, Maître de Recherche

Centre de Recherche Océanologique d'Abidjan, Abidjan, Côte d'Ivoire Coulibaly Safiatou, PhD

Centre de Recherche Océanologique d'Abidjan, Abidjan, Côte d'Ivoire, Département de Génétique et Aquaculture

Tidou Abiba Sanogo, Professeur Titulaire

Université Lorougnon Guédé, Daloa, Côte d'Ivoire Atsé Boua Célestin, Professeur Titulaire

Centre de Recherche Océanologique d'Abidjan, Abidjan, Côte d'Ivoire, Département de Génétique et Aquaculture

Doi:10.19044/esj.2019.v15n21p455 URL:http://dx.doi.org/10.19044/esj.2019.v15n21p455

\section{Resume:}

La lagune Ebrié subit de nombreuses perturbations d'origine anthropique se manifestant depuis ces dernières décénis par une mortalité massive et subite des peuplements ichthyologiques. Afin de mesurer l'impact de ces perturbations, la relation longueur-poids a été estimée pour les principales espèces ichtyologiques de cette lagune. Les spécimens ont été capturés de février 2014 à janvier 2015 à l'aide de filets maillants dans les secteurs IV et V. Après identification, chaque spécimen a été, pesé au gramme près et mesuré au millimètre près. Pour cette étude, 15 espèces appartenant à 11 familles ont été sélectionnées en raison de leur présence sur toute la période de la collecte. Les régressions longueur-poids ont été significatives avec un coefficient de détermination $\left(\mathrm{r}^{2}\right)$ variant entre 0,717 (Ethmalosa fimbriata) et 0,923 (Gerres eucinostomus). Le coefficient d'allométrie b a varié de 2,36 pour Tylochromis jentinki à 3,12 pour Liza falcipinnis. Le coefficient de 
régression de la majorité des espèces capturées $(66,67 \%)$ a été inférieur à 3 . La distribution de fréquence de taille a montré que $92 \%$ des espèces capturées sont de petites tailles avec une taille minimale de $30 \mathrm{~mm}$ pour Gerres nigri et maximale de $370 \mathrm{~mm}$ pour Chrysichthys spp.

Mots clés : Coefficient d'allométrie, relation longueur-poids, lagune Ebrié, Afrique de l'Ouest

\title{
Lenght and Weight Relationship for Fifteen (15) Fish Species Captured in the Ebrié Lagune, Cote d'Ivoire (West Africa)
}

\section{Boni Laurence, PhD}

Unité de Formation et de Recherche des Sciences de l'Homme et de la Société, Département de Géographie, Université Félix Houphouët Boigny, République de Côte d'Ivoire

\section{Nobah Céline Sidonie Koco, Maître de Conférences}

Ecole Normale Supérieure d'Abidjan, Département des Sciences et Technologie, Section Science de la Vie et de la Terre, Abidjan, Côte d'Ivoire

Konan Kouadio Justin, Maître de Recherche

Centre de Recherche Océanologique d'Abidjan, Abidjan, Côte d'Ivoire

\section{Coulibaly Safiatou, PhD}

Centre de Recherche Océanologique d'Abidjan, Abidjan, Côte d'Ivoire,

Département de Génétique et Aquaculture

Tidou Abiba Sanogo, Professeur Titulaire

Université Lorougnon Guédé, Daloa, Côte d'Ivoire

Atsé Boua Célestin, Professeur Titulaire

Centre de Recherche Océanologique d'Abidjan, Abidjan, Côte d'Ivoire,

Département de Génétique et Aquaculture

\begin{abstract}
The Ebrié lagoon undergoes numerous disturbances of anthropic origin manifesting since these last decennis by a massive and sudden mortality of the ichthyological populations. In order to measure the impact of these disturbances, length-weight relationship was estimated for the main ichthyologic species in the lagoon Ebrié. The specimens were collected from February 2014 to January 2015 using gillnets in sectors IV and V. After
\end{abstract}


identification, each sample was weighed to the gram and measured to average millimeter. For this study, 15 species belonging to 11 families were selected because of their presence during the sample period. The length-weight regressions were significant with a coefficient of determination $\left(\mathrm{r}^{2}\right)$ ranging from 0.717 (Ethmalosa fimbriata) to 0.923 (Gerres eucinostomus). The allometry coefficient $\mathrm{b}$ is ranged from 2.36 for Tylochromis jentinki to 3.12 for Liza falcipinnis. The majority of the regression coefficient of the captured species $(66.67 \%)$ was lower than 3. The size frequency distribution showed that $92 \%$ of the captured species are small in size with a minimum size of 30 $\mathrm{mm}$ for Gerres nigri and a maximum of $370 \mathrm{~mm}$ for Chrysichthys spp.

Keywords: Coefficient of allometry, length-weight relationship, Ebrié lagoon, West Africa

\section{Introduction}

La plupart des lagunes de l'Afrique de l'Ouest sont des écosystèmes très productifs qui soutiennent d'importantes activités de pêche. Cependant, la croissance démographique, accentuée par de fréquentes pollutions constitue un réel danger pour leurs ressources.

La Côte d'Ivoire située dans cette zone, a un réseau dense de rivière dont la ressource halieutique annuelle est estimée à 170000 tonnes (PDPA, 2010). Cependant, la production de pêche nationale couvre seulement $20 \%$ des besoins nationaux s'élevant à 300.000 tonnes par an. Cette faible productivité est essentiellement due à la dégradation des écosystèmes aquatiques et aux pêches intensives (Coulibaly, 2012). Depuis des décennies, la qualité de l'eau de la lagune d'Ebrié dépend fortement des activités humaines dues à la croissance démographique rapide et à l'urbanisation incontrôlée. Ce qui implique des conséquences écologiques désastreuses (Akpétou et al, 2010 ; Bamba, 2010 ; Coulibaly et al., 2010). En effet, depuis octobre 1999, une mortalité subite et massive des poissons dans la lagune d'Ebrié a été observée dans la zone lagunaire de Jacqueville et de Dabou au Sud de la Côte d'Ivoire appelée Secteurs IV et $\mathrm{V}$. La périodicité était de cinq à six ans, mais malheureusement, ces dernières années, la fréquence de ce phénomène a augmenté et il en résulte la fermeture de la pêche dans ces secteurs (Hayé et al., 2009; Koffi, 2013). Selon Albaret et Laë (2003), la pression de la pêche et la pollution induisent d'importants changements dans les communautés de poissons. Entre autres, la baisse de la biodiversité, de la taille moyenne des captures, de leur niveau trophique, ce qui nécessite l'utilisation de modèles globaux et analytiques pour la gestion rationnelle de ces ressources. Ces modèles exigent le plus souvent la connaissance de la relation taille-poids des différentes populations. 
Le rapport longueur-poids est une clé d'une importance capitale utilisée dans l'évaluation des pêcheries et en biologie des poissons (Haimovici et Velasco, 2000 ; Da Costa et Araàjo, 2003). En effet, la longueur et le poids peuvent donner des informations sur la composition des stocks, la durée de vie, la mortalité, la croissance et la production (Bolger et Connoly, 1989; King, 1996). Cet outil fournit des informations relatives à la structure et à la fonction des peuplements ichthyologiques (Anderson et Neumann, 1996). Il peut être utilisé pour évaluer l'embonpoint de population de poissons et leur distribution (Bolgerand et Connolly, 1989 ; Safran, 1992) (Kolher et al., 1995).

La présente étude décrit les relations longueur-poids de 15 principales espèces ichtyologiques des secteurs IV et $\mathrm{V}$ du système lagunaire Ebrié subissant les conséquences de la mortalité massive des poissons et celle de la pression des activités de pêche en Côte d'Ivoire.

\section{Materiel et Methodes \\ Zone d'étude}

La lagune Ebrié, avec une superficie de $566 \mathrm{~km}^{2}$ est la plus grande lagune de l'Afrique de l'Ouest. elle est située au Sud de la Côte d'Ivoire $\left(5^{\circ} 13^{\prime} 15^{\prime \prime} \mathrm{N}\right.$ et $\left.4^{\circ} 42^{\prime} 0^{\prime \prime} \mathrm{W}\right)$. Cette lagune a été reliée à l'Océan Atlantique par le Canal de Vridi en 1950 (Figure 1). La lagune Ebrié bénéficie d'un climat de type équatorial de transition incluant 4 saisons annuelles dont deux pluvieuses (avril à juillet et octobre à novembre) et deux sèches (décembre à mars et août à septembre). La communication constante avec l'océan produit des caractéristiques typiques de l'estuaire, dans le secteur IV. En revanche, le secteur V est oligohalin et caractérisé par des eaux stables et homogènes toute l'année avec une salinité variant de 0 à $3 \%$ (Ecoutin et Albaret, 2003).

\section{Collecte des données}

Les échantillons de poissons ont été recueillis mensuellement de février 2014 à janvier 2015 dans les zones allant de Songon (secteur IV) à Jacqueville (secteur V) à l'aide de plusieurs batteries de filet maillant (6 mm à $60 \mathrm{~mm}$ maille étirée). Ces filets ont été posés le soir aux environs de $17 \mathrm{~h}$ et relevés le lendemain à partir de $7 \mathrm{~h}$. Les 15 spécimens récoltés ont été identifiés à l'aide des clés d'identification de Lévêque et al. $(1990,1992)$. Chaque individu capturé a été pesé à $0,01 \mathrm{~g}$ près et mesuré (LS) au mm près. Seules les espèces dont la présence a été notée sur toutes les stations pendant toute la période de collecte ont été utilisées dans cette étude.

\section{Traitement et analyse statistique des données}

Pour caractériser la structure de la communauté des poissons de la zone d'étude, la distribution des fréquences de tailles de l'ensemble des individus 
capturés a été établie à partir des longueurs standards. Cela a permis de déterminer les tailles dominantes dans les captures.

Les relations longueur-poids ont été établies pour les 15 espèces échantillonnés selon la formule suivante :

$\mathbf{P}=\mathbf{a} \mathbf{L} \mathbf{S}^{\mathbf{b}}$

avec $\mathrm{P}=$ masse du poisson en $(\mathrm{g}), \mathrm{LS}=$ longueur standard du poisson en $(\mathrm{mm})$, $\mathrm{a}=$ constante et $\mathrm{b}=$ coefficient d'allométrie.

Les paramètres $\mathrm{a}$ et $\mathrm{b}$ ont été estimés après la transformation de la fonction linéaire précédente en une fonction logarithmique de formule :

$\log \mathbf{P}=\log \mathbf{a}+\operatorname{blog} \mathrm{L}$

Afin de vérifier si la valeur de b est sensiblement différente de 3 , le test de t-Student a été utilisé $(\mathrm{p}=0,05)$. Le coefficient d'allométrie $\mathrm{b}$ varie entre 2 et 4 , mais il est le plus souvent proche de 3. Quand $b=3$, il y a isométrie de croissance (et la densité spécifique de l'animal ne change pas). Si b $>3$, l'allométrie est majorante, le poisson grossit plus vite qu'il ne grandit. Si b < 3, l'allométrie est minorante, le poisson grandit plus vite qu'il ne grossit. Le coefficient de détermination $\mathrm{r}^{2}$ a été utilisé comme un indicateur du degré de corrélation entre la longueur et la masse.

\section{Resultats}

\section{Distribution des fréquences de taille}

A l'analyse de la distribution des fréquences de taille (Figure 3), cinq classes d'individus ont été rencontrées : les individus de tailles inférieures à $80 \mathrm{~mm}(12 \%)$, ceux de tailles comprises entre $80 \mathrm{~mm}$ et $100 \mathrm{~mm}(27 \%)$, ceux de tailles oscillant entre $101 \mathrm{~mm}$ et $150 \mathrm{~mm}(47 \%)$, ceux de tailles variant de $151 \mathrm{~mm}$ à $180 \mathrm{~mm}(9 \%)$ et ceux de tailles au-delà de $180 \mathrm{~mm}(5 \%)$.

Le groupe d'espèces Chrysichthys spp. a été le plus collecté au cours de cette étude $(\mathrm{n}=700)$ avec des tailles variant de $35 \mathrm{~mm}$ à $310 \mathrm{~mm}$. Sur l'ensemble des spécimens capturés, environ $90 \%$ des individus ont des tailles comprises entre $80 \mathrm{~mm}$ et $150 \mathrm{~mm}$ contre $7 \%$ d'individus de tailles oscillant entre $152 \mathrm{~mm}$ et $260 \mathrm{~mm}$. Pour les autres espèces telles que Gerres nigri, Eucinostomus melanopterus, Trachinotus teraia, Ethmalosa fimbriata, l'hybride Tilapia zilli x Tilapia guineensis, Sarotherodon melanoteron, Tylochromis jentinki, Citharichthys stampfii et Pomadassys jubelini, les 2/3 des individus ont des tailles comprises entre $100 \mathrm{~mm}$ et $150 \mathrm{~mm}$. La majorité des spécimens de Monodactylus sebae ont des tailles inférieures à $80 \mathrm{~mm}$. Pour Elops lacerta, Liza falcipinis et Cynoglossus senegalensis, le plus petit individu a une taille supérieure à $100 \mathrm{~mm}$. Toutefois, la taille de $50 \%$ des 
individus est comprise entre $100 \mathrm{~mm}$ et $150 \mathrm{~mm}$ chez E. lacerta contre $38 \%$ chez L. falcipinis. Pour C. senegalensis, la taille du plus petit individu est de $122 \mathrm{~mm}$.

\section{Relation longueur-poids}

Les résultats de la relation longueur-poids de 15 espèces de poissons constituant un échantillon de 3410 spécimen appartenant à 11 familles sont indiqués dans le Tableau 1. Les familles avec le plus grand nombre d'espèces sont les Cichlidae $(n=5)$, les Gerridae $(n=2)$. Les autres familles monospecifiques, Carangidae, Paralichthydae, Cynoglossidae, Elopidae, Mugilidae, Monodactylidae, Clupeidae, Haemulidae, Claroteidae). Le nombre de spécimens varie de 48 individus pour $M$. sebae à 700 individus pour Chrysichthys spp. La longueur standard varie de $30 \mathrm{~mm}$ (G. nigri) à $370 \mathrm{~mm}$ (Chrysichthys spp.) et la masse de $3 \mathrm{~g}$ (Cytharichthys stamflii ; Hybride T. zilli $x$ T. guineensis, T. jentinki) à $843 \mathrm{~g}$ (chrysichthys spp.).

Les valeurs du coefficient de détermination $\left(\mathrm{r}^{2}\right)$ oscillent entre 0,717 pour E. fimbriata et 0,923 pour $G$. eucinostomus. La valeur estimée du coefficient b varie de 2,36 (T. jentinki) à 3,12 (L. falcipinnis). La majorité des espèces ont un coefficient de régression inférieur à 3 (Figure 2).

En termes de croissance, les résultats ont montré que le coefficient d'allométrie (b) de 3 espèces, Trachinotus teraia, l'hybride Tilapia guineensis $\times$ Tilapia zillii et Pomadasys jubelini est égal à 3 (test-t de Student: $\mathrm{p}=0,05$ ), attestant que la croissance est isométrique (Tableau 2). En revanche, 12 espèces $(80 \%)$ ont un coefficient $b$ significativement différent de 3 (test-t de Student: $\mathrm{p}<0,05)$. Au nombre de ces espèces, 10 espèces présentent une croissance allométrique minorante (b < 3). Il s'agit de Cytharichthys stamflii, Cynoglossus senegalensis, Elops lacerta, Gerres nigri, Hemichromis fasciatus, Sarotherodon melanotheron, Tylochromis jentinki, Monodactylus sebae, Ethmalosa fimbriata et Chrysichthys maurus. En revanche, 2 espèces, Eucinostomus menaleptorus, liza falcipinnis ont une croissance allométrique majorante $(\mathrm{b}>3)$.

\section{Discussion}

Les individus échantillonnés dans cette étude ont des tailles oscillant entre $80 \mathrm{~mm}$ et $260 \mathrm{~mm}$ (LS) avec la présence d'une forte proportion de spécimens de petites tailles. La fréquence de distribution de taille présente une allure gaussienne et montre que le plus grand nombre d'individus collectés est concentré entre 80 et $150 \mathrm{~mm}$, soit $90 \%$ de la population. Cela porte à croire que cette zone de la lagune Ebrié est utilisée comme nurserie par les différentes espèces de poissons. La diminution des tailles moyennes des individus pourrait traduire la surexploitation des stocks. Selon Albaret et Laë (2003), cette partie de la lagune est pleinement exploitée par une pêche professionnelle 
reposant à la fois sur l'utilisation d'engins individuels et collectifs. Ces poissons soumis à cette pression pourraient présenter des adaptations écophysiologiques en rapport avec la croissance ou la reproduction telle que la maturité sexuelle précoce, le nanisme... . Par ailleurs depuis 1999, d'importantes mortalités ont été enregistrées généralement en début de la grande saison des pluies dans cette zone (Hayé et al., 2009). Ces mortalités ont atteint un pic en mai 2013 avec la perte de plusieurs centaines de tonnes de poissons (Koffi, 2013). Le repeuplement de cette zone à partir des autres secteurs de la lagune, est une hypothèse qui pourrait expliquer en partie la caractérisation de ces peuplements ichtyologiques. En outre, la composition des captures pourrait être le reflet des engins et des techniques de pêche utilisées dont la sélectivité, en fonction des caractéristiques morphologiques ou comportementales des individus, peut jouer en faveur de la capture d'une taille donnée.

Le coefficient d'allométrie a varié de 2,36 ( $T$. jentinki) à 3,12 ( $L$. falcipinnis) avec une moyenne de 2,707 $\pm 1,063$. Cette valeur est significativement différente de 3 (test-t de Student : p < 0.05). En outre, le coefficient d'allométrie est significativement inférieur à 3 pour la majorité des espèces (Figure 2). Les valeurs de ce coefficient pour E. melanopterus $(3,08)$, pour l'hybride $T$. zillii $x T$. guineensis $(2,94)$ et pour $P$. jubelini $(2,96)$ ne sont pas significativement différentes de 3 (test-t de Student : p =0,05), indiquant une croissance isométrique. En revanche, T. teraia et $L$. falcipinis, ont une croissance allométrique positive se traduisant par un coefficient $\mathrm{b}$ supérieur à 3 (test-t de Student : p < 0,05). Ce qui indique une meilleure croissance en poids qu'en longueur (Micha, 1973 ; Ricker, 1980). Neuf (9) espèces (C. stampfii, C. senegalensis, E. lacerta, G. nigri, H. fasciatus ,S. melanotheron, $M$. sebae, E. fimbriata, Chrysichthys spp.) présentent une allométrie inférieure à 3 (test- $t$ de Student $: p<0,05$ ). Les valeurs de $b$ pour toutes les espèces sont dans les limites déclarées (2-4) par Tesch (1971) et Bagenal et Tesch (1978) pour la plupart des poissons. Cependant, pour la plupart de ces espèces, la valeur du coefficient $b$ indique une allométrie négative. Cette gamme de valeur b est similaire à ceux des travaux de Tah et al. (2012) pour 36 espèces de deux réservoirs tropicaux en Côte d'Ivoire, de Konan et al. (2007) pour 57 espèces de petits fleuves côtiers dans le Sud-Est de la Côte d'Ivoire et de Ecoutin et Albaret (2003) pour 52 espèces de lagunes et estuaires en Afrique de l'Ouest. Ces différents auteurs ont obtenu des coefficients d'allométrie respectifs allant de 2,17 à 3,42, de 2,21 à 3,73 et de 2,46 à 3,47. Selon Biswas (1993), la variation de $b$ dans les différentes régions est due aux fluctuations saisonnières des paramètres environnementaux, aux conditions physiologiques du poisson au moment de la collecte, au sexe, au développement des gonades et aux conditions nutritives dans l'environnement des poissons. 
Le coefficient de corrélation $\left(\mathrm{r}^{2}\right)$ pour la relation longueur-poids de la majorité des poissons a varié de 0,717 à 0,979 , indiquant que ces individus ont une croissance pondérale faible. Ces résultats sont similaires aux études antérieures portant sur les mêmes espèces de poissons (Koffi et al., 2014 ; Ecoutin et Albaret, 2003).

\section{Conclusion}

En définitive, 15 espèces ichtyologiques appartenant à 11 familles identifiées pour cette étude présentent des régressions longueur-poids significatives avec un coefficient de détermination $\left(\mathrm{r}^{2}\right)$ variant entre $0,717(E$. fimbriata) et 0,923 (G. eucinostomus). Le coefficient d'allométrie b a varié entre 2,36 pour $T$. jentinki et 3,12 pour L. falcipinnis. Cette étude montre par ailleurs une diminution des tailles moyennes des individus qui pourrait traduire la surexploitation des stocks et des adaptations écophysiologiques en rapport avec la croissance ou la reproduction. Remerciement : Ce travail a été réalisé dans le cadre de l'étude de l'effet des changements environnementaux sur la vulnérabilité des ressources halieutiques dans la zone allant de Jacqueville à Dabou (Lagune Ebrié). Ce projet a été financé par le FIRCA. Les auteurs tiennent a remercié toutes les personnes qui ont participé à la collecte des données sur le terrain et les chercheurs qui ont été d'un apport appréciable pour la conduite des travaux.

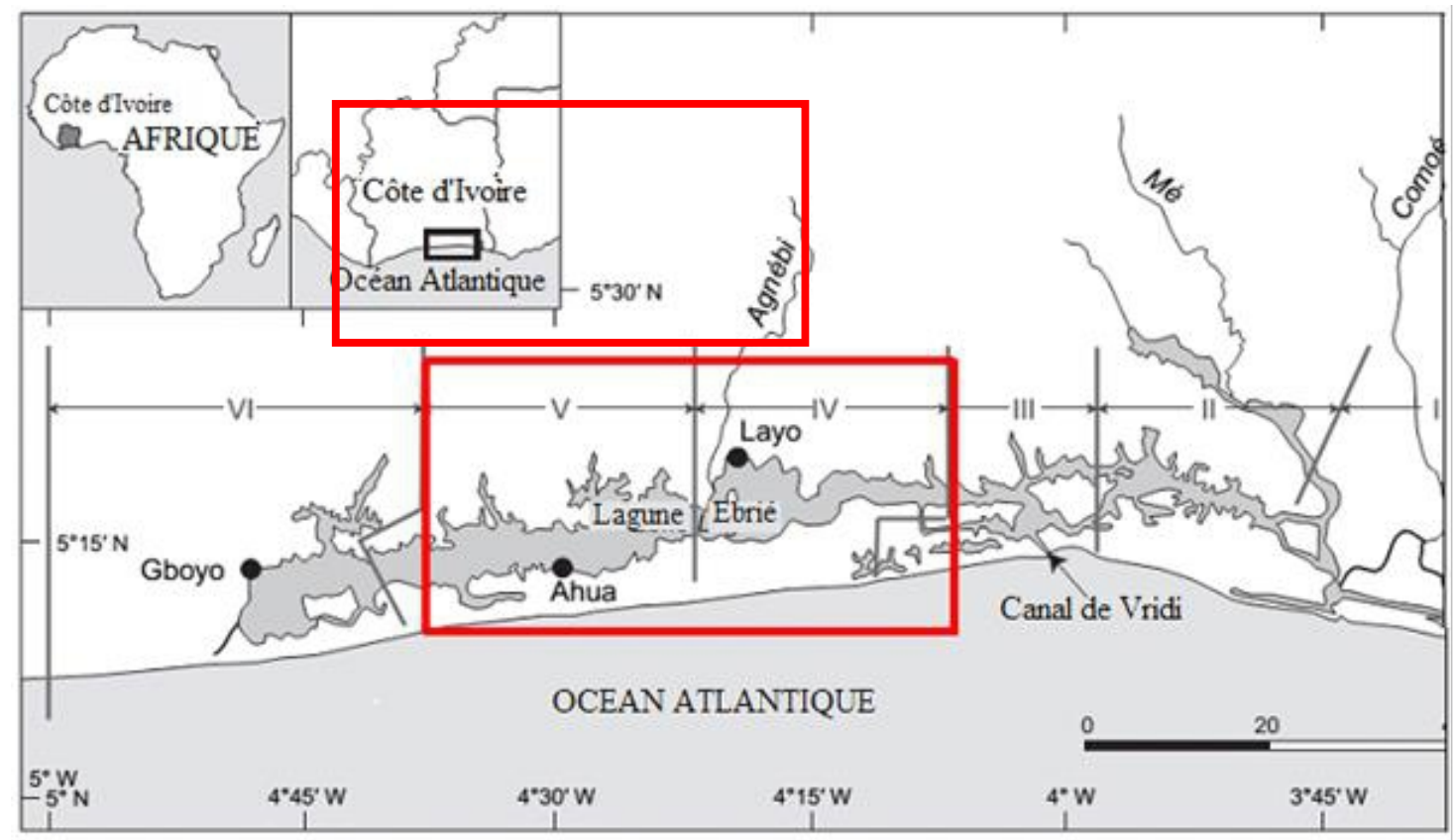

Figure 1 : Situation géographique de la lagune Ebrié et localisation de la zone d'étude en encadré. 


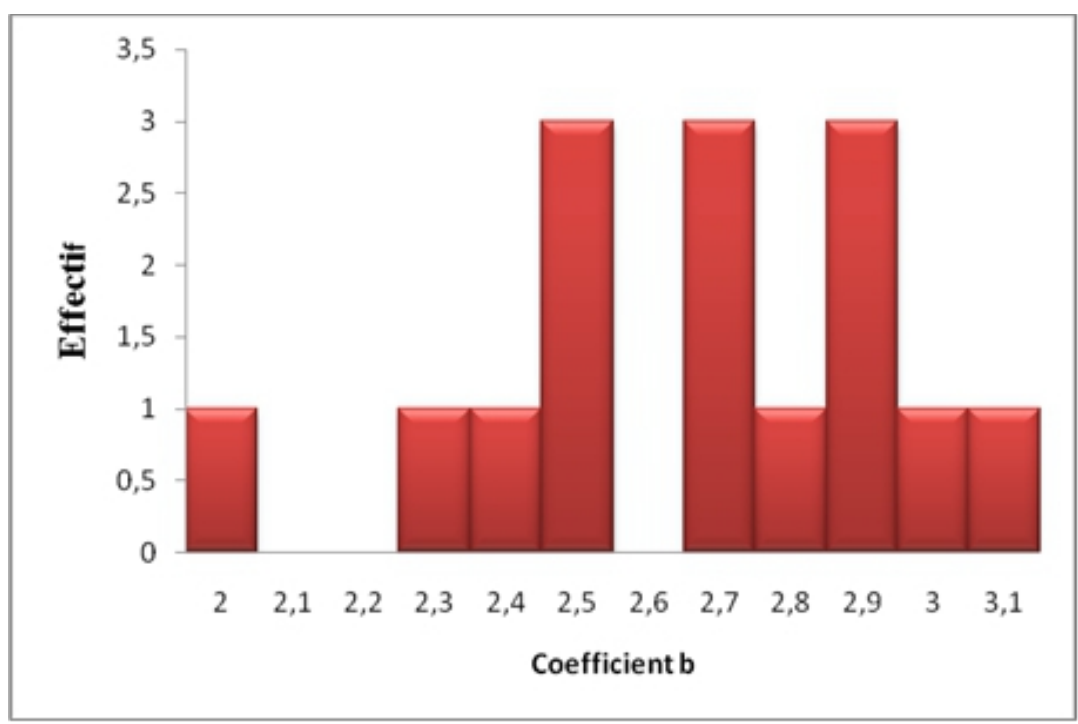

Figure 3 : distribution du coefficient d'allométrie b pour les 15 espèces ichtyologiques de la lagune Ebrié. 
Tableau1 : Relation taille -poids, coefficient d'allometrie et type de croissance de 15 espèces ichtyologiques capturées dans la zone IV et V de la lagune Ebrié.

Min : minimum ; Max : maximum ; a et $\mathbf{b}$ : Paramètres de croissance ; $\mathbf{S E}:$ erreur standard ; $\mathbf{r}^{2}$ : coefficient de détermination ; A : allométrie ; I : Isométrie

\begin{tabular}{|c|c|c|c|c|c|c|c|c|c|c|c|}
\hline \multirow[b]{2}{*}{ Famille } & \multirow[b]{2}{*}{ Espèces } & \multicolumn{3}{|c|}{ Longueur (mm) } & \multicolumn{3}{|c|}{$\operatorname{Masse}(g)$} & \multirow[b]{2}{*}{$\mathbf{b}$} & \multirow[b]{2}{*}{ SE (b) } & \multirow[b]{2}{*}{$\mathbf{r}^{2}$} & \multirow[b]{2}{*}{ Allométrie } \\
\hline & & Nombre & Min & Max & Min & Max & $\mathbf{a}$ & & & & \\
\hline Carangidae & Trachinotus teraia & 194 & 46 & 320 & 19 & 224 & 0,0106 & 2,990 & 0,161 & 0,918 & $\mathrm{~A}^{+}$ \\
\hline Paralichthyidae & Cytharichthys stamflii & 59 & 39 & 158 & 3 & 83 & 0,0237 & 2,500 & 0,138 & 0,782 & $\mathrm{~A}^{-}$ \\
\hline Cynoglossidae & Cynoglossus senegalensis & 82 & 50 & 390 & 8 & 181 & 0,0462 & 2,016 & 0,0823 & 0,774 & $\mathrm{~A}^{-}$ \\
\hline Eleopidae & Elops lacerta & 223 & 101 & 265 & 14 & 188 & 0,011 & 2,77 & 0,041 & 0,851 & $\mathrm{~A}^{-}$ \\
\hline Gerridae & Eucinostomus melanopterus & 210 & 60 & 152 & 1 & 89 & 0,0086 & 3,079 & 0,0290 & 0,923 & I \\
\hline Gerridae & Gerres nigri & 260 & 30 & 181 & 4 & 87 & 0,017 & 2,742 & 0,0153 & 0,878 & $\mathrm{~A}^{-}$ \\
\hline Cichlidae & Hemichromis fasciatus & 193 & 55 & 180 & 5 & 81 & 0,016 & 2,770 & 0,024 & 0,896 & $\mathrm{~A}^{-}$ \\
\hline Cichlidae & $\begin{array}{l}\text { Hybride Tilapia zilli } \quad x \quad T . \\
\text { guineensis }\end{array}$ & 382 & 55 & 190 & 3 & 225 & 0,013 & 2,936 & 0,048 & 0,918 & I \\
\hline Cichlidae & Sarotherodon melanotheron & 98 & 70 & 186 & 13 & 245 & 0,017 & 2,829 & 0,091 & 0,899 & $\mathrm{~A}^{-}$ \\
\hline Cichlidae & Tylochromis jentinki & 399 & 40 & 190 & 3 & 187 & 0,0167 & 2,361 & 0,024 & 0,817 & $\mathrm{~A}^{-}$ \\
\hline Mugilidae & Liza falcipinis & 71 & 100 & 283 & 16 & 300 & 0,0064 & 3,12 & 0,338 & 0,855 & $\mathrm{~A}^{+}$ \\
\hline Monodactylidae & Monodactylus sebae & 48 & 40 & 105 & 4 & 77 & 0,031 & 2,591 & 0,113 & 0,877 & $\mathrm{~A}^{-}$ \\
\hline Clupeidae & Ethmalosa fimbriata & 375 & 70 & 160 & 9 & 142 & 0,024 & 2,51 & 0,0169 & 0,717 & $\mathrm{~A}^{-}$ \\
\hline Haemulidae & Pomadasys jubelini & 116 & 70 & 235 & 7 & 350 & 0,010 & 2,96 & 0,160 & 0,879 & $\mathrm{I}$ \\
\hline Claroteidae & Chrysichthys sp & 700 & 35 & 370 & 4 & 843 & 0,029 & 2,45 & 0,19 & 0,782 & $\mathrm{~A}^{-}$ \\
\hline
\end{tabular}



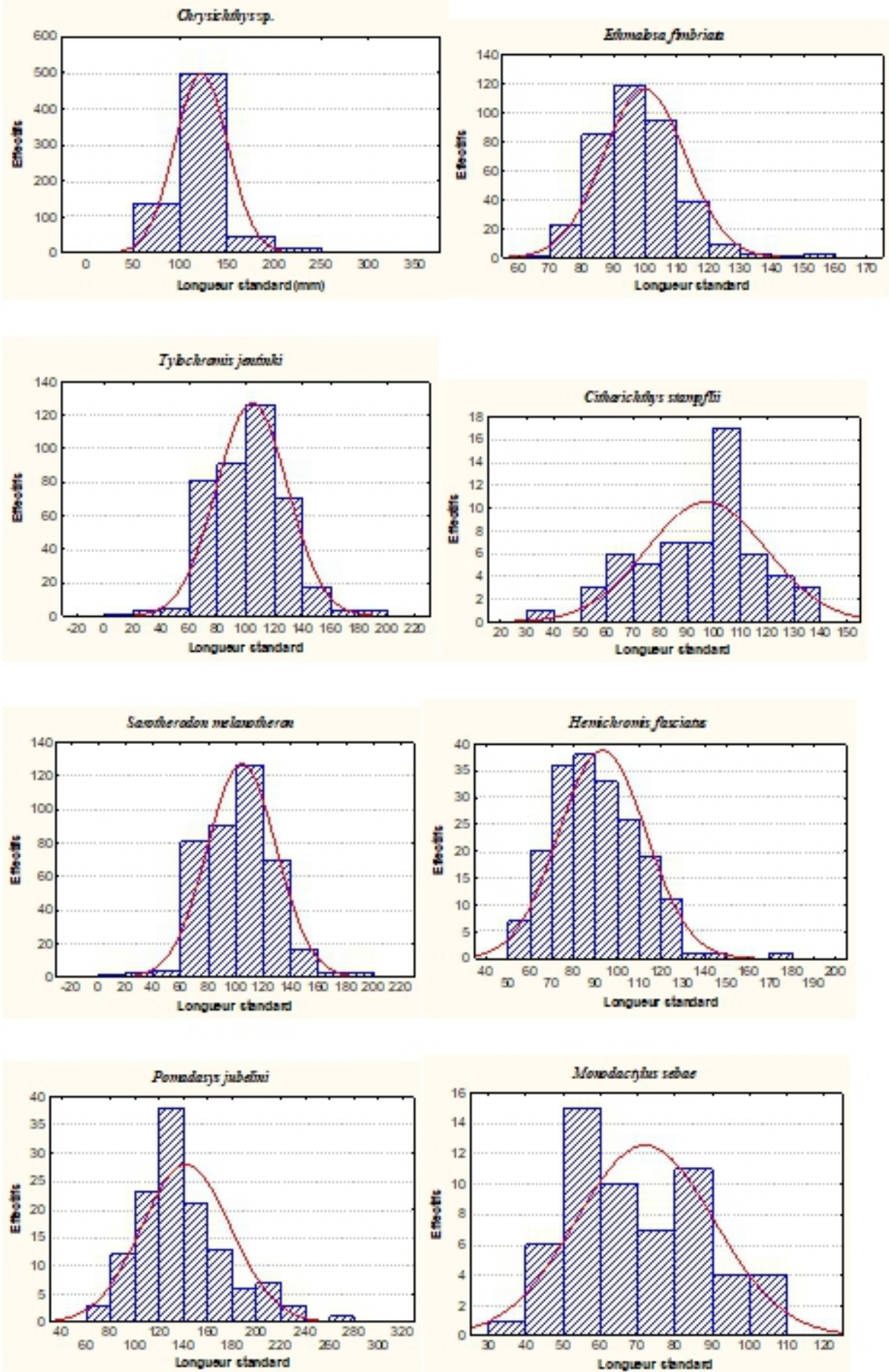

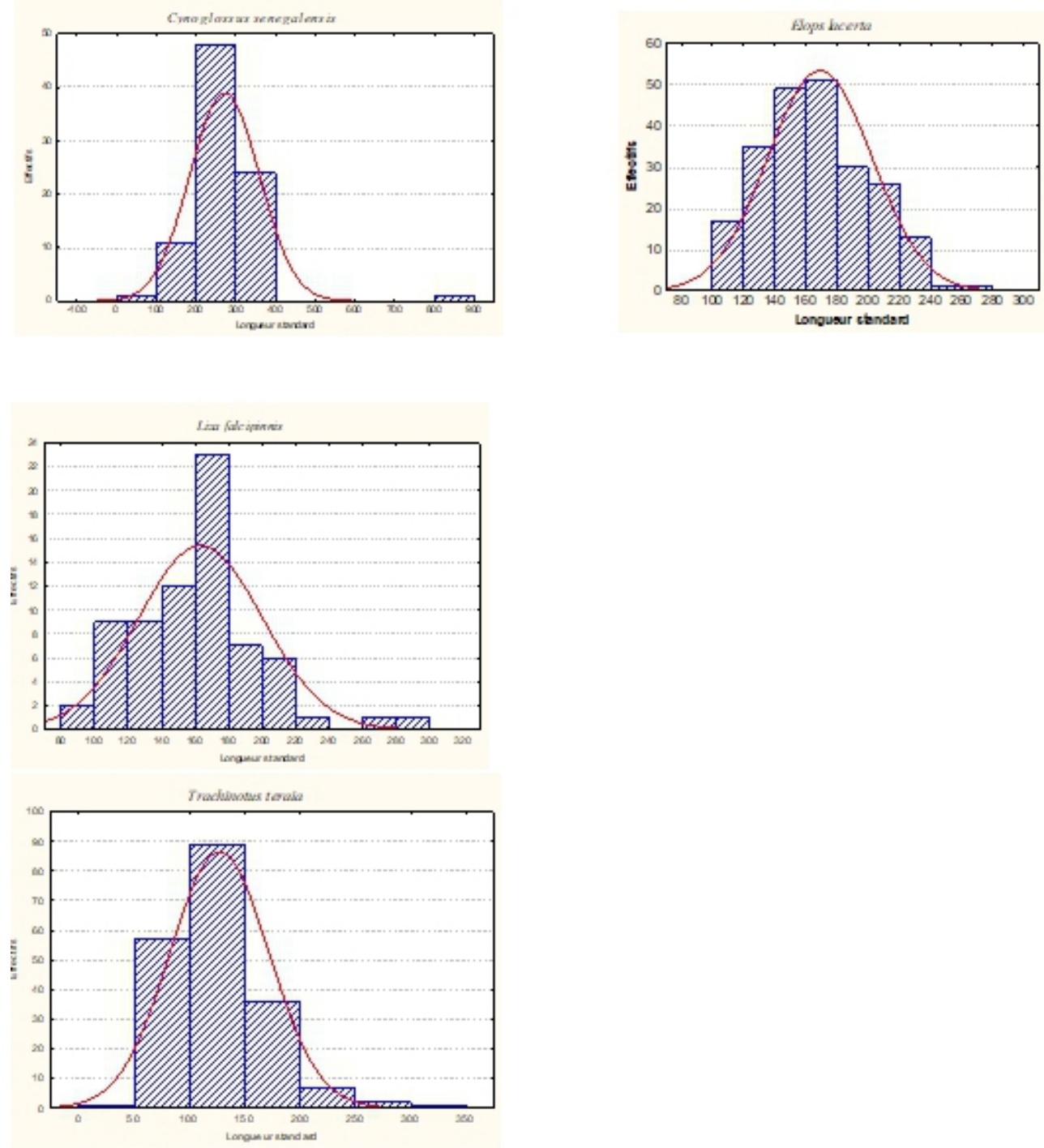

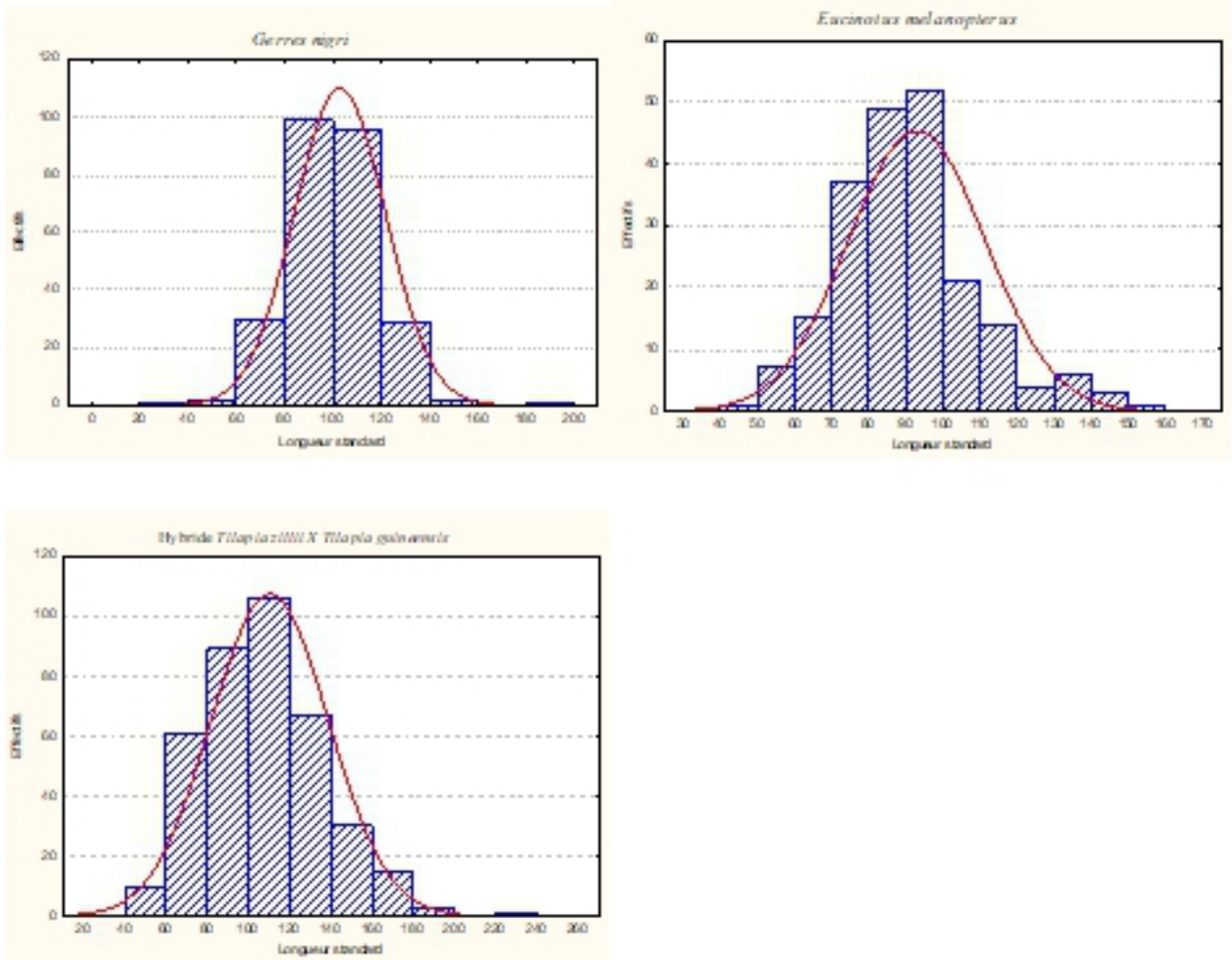

Figure 2: Histogramme des fréquences de tailles des différentes espèces

\section{References:}

1. Albaret, J.J. \& Laë, R. (2003). Impact of fishing on fish assemblages in tropical lagoons: The example of the Ebrie lagoon, West Africa. Aquatic. Liv.. Res, 16: 1-9.

2. Anderson, O.R. \& Neumann, R. M. (1996): Length, weight and associated structural indices. In Nielsen, L.A. \& Johnson, D.L. (Eds.) Fisheries techniques. Bethesda, Amer. Fish. Soc., 447-482.

3. Bagenal, T.B. \& Tesch, F.W. (1978). Age and Growth. In: Bagenal, T. (Ed.), Methods for Assessment of Fish Production in Freshwater. IBP Handbook 3. Blackwell Scientific Publications, Oxford, UK, pp: 101136.

4. Bolger, T. \& Connoly P.L. (1989). The selection of suitable indice for the measurement and analysis of fish condition. J. Fish .Biol., 34: 17?182.

5. Biswas, S.P.L (1993). Manual of methods in fish biology South Asian Publisher. Pages?

6. Clariidae) dans le delta de l'Ouémé au Bénin (Afrique de l'Ouest). Research Journal of Biological Sciences, 2(2): 157-167. 
7. Da Costa, S. \& Araàjo, F.G. (2003). Length-weight relationship and condition factor of Micropogonias furnieri (Desmarest) (Perciformes, Sciaenidae) in the Sepetiba Bay, Rio de Janeiro State, Brazil. Revista Brasileira de Zoologia, 20(4): 685-690.

8. Ecoutin, J.M, Albaret, J.J. (2003). Rélation longueur-poids pour 52 espèces de poissons des estuaires et lagunes de l'Afrique de l'Ouest. Cybium, 27(1): 3-9.

9. Fagade, S.O. (1983). The biology of Chromidotilapia guntheri from a small lake. Archiv of Hydrobiology, 97: 60-72.

10. Haimovici, M. \& Valasco, G. (2000). Lenght-weight relationship of marine fishes from southern Brazil. The ICLARM Quaterly 23(1):1416.

11. Hayé C. V., Dongui B. K., Pellerin J. et Trokourey A., 2009. Pollution evaluation in the estuary bay of Biétri (Abidjan, Côte d'Ivoire). Journal of Oceanography, Research and Data, 2: 1-11.

12. King, R.P. (1991). The biology of Tilapia mariae Boulenger 1899 (Perciformes: Cichlidae) in a Nigerian rainforest stream. Ph.D. Thesis, University of Port Harcourt, Nigeria.

13. King, R.P. (1996). Length-weight relatioship of nigerian coastal water fishes. Fishbyte, 19 (4) 54-58.

14. Koffi, K.B., Berté, S. \& Koné, T. (2014). Length-weight Relationships of 30 Fish Species in Aby Lagoon, Southeastern Côte d'Ivoire, Current Research Journal of Biological Sciences 6(4): 173-178.

15. Koffi P., 2013. Des poissons toxiques sur le marché ? Le Nouveau Réveil $\mathrm{N}^{\circ}$ 3408, Abidjan. 6p. Konan, A.K.F., Ouattara, M., Ouattara, A. \& Gourène, G. (2007). Weight-length relationship of 57 fish species of the coastal rivers in south-eastern of Ivory Coast. Ribarstvo, 65(2): 49-60.

16. Konan, Y.A., Siaka, O., Koné, T., Bamba M. \& Koné, I. (2013). Caractéristiques de la reproduction de Thysochromis ansorgii (Pisces, Cichlidae) dans la forêt des marais Tanoé-Ehy (Côte d'Ivoire). Journal of Applied Biosciences, 71: 5715- 5727.

17. Kouadio, K.N., Diomandé, D., Ouattara, A., Koné, Y.J.M. \& Gourène, G. (2008). Taxonomic diversity and structure of benthic macroinvertebrates in Aby lagoon (Ivory Coast, West Africa). Pakistan Journal of Biological Sciences, 11(18): 2224-2230.

18. Lalèyé, P.A. (2006). Length-weight relationships of fishes from the Queme River in Benin (West Africa). Journal of Applied Ichthyology, 22: 330-333.

19. Lévêque, C., Paguy, D., Teugels, G.G. (1990): Faune des poissons d'eaux douces et saumâtres de l'Afrique de l'ouest. Tome I. Faune tropicale, XXVIII, MRAC-Tervuren / ORSTOM-Paris, 1-384. 
20. Lévêque, C., Paguy, D., Teugels, G. G. (1992): Faune des poissons d'eaux douces et saumâtres de l'Afrique de l'ouest. Tome II. Faune tropicale, XXVIII, MRAC-Tervuren / ORSTOM-Paris, 385-902.

21. Tah, L., Gooré Bi, G. \& Da Costa, K.S. (2012). Length-weight relationships for 36 freshwater fish species from two tropical reservoirs: Ayamé I and Buyo, Côte d'Ivoire. International Journal of Tropical Biology, 60(4): 1847-1856.

22. Tesch, F.W. (1971). Age and Growth. In: Ricker, W.E. (Ed.), Methods for Assessment of Fish Production in Freshwaters. IBP Handbook 3. Blackwell Scientific Publications, Oxford, UK, pp: 93-123. 